Article

\title{
Outcome of Relapsed or Refractory FLT3-Mutated Acute Myeloid Leukemia before Second-Generation FLT3 Tyrosine Kinase Inhibitors: A Toulouse-Bordeaux DATAML Registry Study
}

\author{
Sarah Bertoli 1,2,3,*,+, Pierre-Yves Dumas 4,5,6,+, Emilie Bérard 7,8, Laetitia Largeaud 2,3,9, \\ Audrey Bidet ${ }^{10}$, Eric Delabesse ${ }^{2,3,9}$, Suzanne Tavitian ${ }^{1}$, Noémie Gadaud ${ }^{1}$, Thibaut Leguay ${ }^{4}$, \\ Harmony Leroy ${ }^{4}\left(\mathbb{D}\right.$, Jean-Baptiste Rieu ${ }^{9}$, Jean-Philippe Vial ${ }^{10}{ }^{1}$, François Vergez ${ }^{2,3,9}$, \\ Nicolas Lechevalier ${ }^{10}{ }^{D}$, Isabelle Luquet ${ }^{9}$, Emilie Klein ${ }^{10}$, Audrey Sarry ${ }^{1}$, \\ Anne-Charlotte De Grande ${ }^{4}$, Christian Récher $1,2,3, \ddagger$ and Arnaud Pigneux $4,5,6, \ddagger$
}

1 Service d'Hématologie, Centre Hospitalier Universitaire de Toulouse, Institut Universitaire du Cancer de Toulouse-Oncopole, 31059 Toulouse, France; tavitian.suzanne@iuct-oncopole.fr (S.T.); gadaud.noemie@iuct-oncopole.fr (N.G.); sarry.audrey@iuct-oncopole.fr (A.S.); recher.christian@iuct-oncopole.fr (C.R.)

2 University Toulouse III Paul Sabatier, 31000 Toulouse, France; largeaud.laetitia@iuct-oncopole.fr (L.L.); delabesse.eric@iuct-oncopole.fr (E.D.); vergez.francois@iuct-oncopole.fr (F.V.)

3 Cancer Research Center of Toulouse, UMR1037 INSERM, ERL5294 CNRS, 31000 Toulouse, France

4 CHU Bordeaux, Service d'Hématologie Clinique et de Thérapie Cellulaire, F-33000 Bordeaux, France; pierre-yves.dumas@u-bordeaux.fr (P.-Y.D.); thibaut.leguay@chu-bordeaux.fr (T.L.); leroy.harmony@gmail.com (H.L.); anne-charlotte.de-grande@chu-bordeaux.fr (A.-C.D.G.); arnaud.pigneux@chu-bordeaux.fr (A.P.)

5 Université de Bordeaux, 33000 Bordeaux, France

6 Institut National de la Santé et de la Recherche Médicale, U1035, 33000 Bordeaux, France

7 Service d'Epidémiologie, Centre Hospitalier Universitaire de Toulouse, 31000 Toulouse, France; emilie.berard@univ-tlse3.fr

8 UMR 1027, INSERM-Université de Toulouse III, 31000 Toulouse, France

9 Laboratoire d'Hématologie, Centre Hospitalier Universitaire de Toulouse, Institut Universitaire du Cancer de Toulouse-Oncopole, 31059 Toulouse, France; rieu.jeanbaptiste@iuct-oncopole.fr (J.-B.R.);

luquet.isabelle@iuct-oncopole.fr (I.L.)

10 CHU Bordeaux, Laboratoire d'Hématologie Biologique, F-33000 Bordeaux, France; audrey.bidet@chu-bordeaux.fr (A.B.); jean-philippe.vial@chu-bordeaux.fr (J.-P.V.); nicolas.lechevalier@chu-bordeaux.fr (N.L.); emilie.klein@chu-bordeaux.fr (E.K.)

* Correspondence: bertoli.sarah@iuct-oncopole.fr; Tel.: +33-531-156-306; Fax: +33-531-156-314

+ These authors contributed equally to this work.

$\ddagger$ These authors contributed equally to this work.

Abstract: A recent phase 3 trial showed that the outcome of patients with relapsed/refractory (R/R) FLT3-mutated acute myeloid leukemia (AML) improved with gilteritinib, a single-agent second-generation FLT3 tyrosine kinase inhibitor (TKI), compared with standard of care. In this trial, the response rate with standard therapy was particularly low. We retrospectively assessed the characteristics and outcome of patients with R/R FLT3-mutated AML included in the Toulouse-Bordeaux DATAML registry. Among 347 patients who received FLT3 TKI-free intensive chemotherapy as first-line treatment, 174 patients were refractory $(n=48,27.6 \%)$ or relapsed $(n=126$, $72.4 \%)$. Salvage treatments consisted of intensive chemotherapy $(n=99,56.9 \%)$, azacitidine or low-dose cytarabine $(n=9,5.1 \%)$, other low-intensity treatments $(n=17,9.8 \%)$, immediate allogeneic stem cell transplantation $(n=4,2.3 \%)$ or best supportive care only $(n=45,25.9 \%)$. Among the 114 patients who previously received FLT3 TKI-free intensive chemotherapy as first-line treatment 
(refractory, $n=32,28.1 \%$; relapsed, $n=82,71.9 \%$ ), the rate of CR (complete remission) or CRi (complete remission with incomplete hematologic recovery) after high- or low-intensity salvage treatment was $50.0 \%$, with a bridge to transplant in $34.2 \%(n=39)$ of cases. The median overall survival (OS) was 8.2 months (interquartile range, 3.0-32); 1-, 3- and 5-year OS rates were $36.0 \%$ (95\%CI: $27-45), 24.7 \%$ (95\%CI: 1-33) and 19.7\% (95\%CI: 1-28), respectively. In this real-word study, although response rate appeared higher than the controlled arm of the ADMIRAL trial, the outcome of patients with R/R FLT3-mutated AML remains very poor with standard salvage therapy.

Keywords: acute myeloid leukemia; FLT3-ITD mutation; FLT3-TKD mutation; primary induction failure; refractory; relapse; tyrosine kinase inhibitors; gilteritinib

\section{Introduction}

FMS-like tyrosine kinase 3 (FLT3) is a class III receptor tyrosine kinase expressed in early hematopoietic stem and progenitor cells that regulates their proliferation and differentiation [1]. FLT3-activating mutations occur in approximately $30 \%$ of patients with acute myeloid leukemia (AML) and, as such, are among the most frequent mutations found in AML [2], either as in-frame internal tandem duplications (ITD) within the juxtamembrane region or as missense point mutations in the tyrosine kinase domain (TKD) $[3,4]$. FLT3-ITD (but not FLT3-TKD) mutations confer a poor prognosis in AML, especially when NPM1 is not co-mutated and the allelic FLT3-ITD/wild-type ratio is high; such mutations are usually conserved at relapse and have therefore emerged as a relevant therapeutic target [5,6]. FLT3 tyrosine kinase inhibitors have been developed for several years, and the type I FLT3 inhibitor (i.e., that has activity against FLT3-ITD and TKD mutations) midostaurin was approved in 2017 for the treatment of newly diagnosed FLT3-mutated AML, in combination with intensive chemotherapy [7]. Furthermore, several second-generation FLT3 inhibitors, such as quizartinib, gilteritinib and crenolanib, have demonstrated single-agent activity that can lead to complete or near-complete remission [8-10].

The ADMIRAL phase 3 trial, designed for relapsed/refractory (R/R) FLT3-mutated AML patients, recently demonstrated the superiority of gilteritinib as single agent over the control treatment arm [8], which was determined by investigators prior to 2:1 randomization between mitoxantrone, etoposide, cytarabine (MEC), fludarabine, cytarabine, granulocyte colony-stimulating factor, idarubicin (FLAG-IDA), azacitidine (AZA) or low-dose cytarabine (LDAC). These regimens are recognized as acceptable salvage regimens in this situation, although other combinations based on intermediate- or high-dose cytarabine or even single-agent cytarabine are also widely used [11]. In the ADMIRAL trial, overall survival (OS) was significantly improved in the gilteritinib arm compared to the control arm with a hazard ratio (HR) of 0.64 (95\%CI: 0.49-0.83; $p<0.001$ ). The median OS was 9.3 months in the gilteritinib arm and 5.6 months in the control arm. The complete remission and complete remission with incomplete hematologic recovery rates were $21.1 \%$ and $25.5 \%$ in the gilteritinib arm vs. $10.5 \%$ and $4.8 \%$ in the standard arm [8].

The aim of our study was to describe the characteristics, treatments and outcome of $R / R$ FLT3-mutated AML patients treated in a routine setting before the approval of second-generation FLT3 inhibitors in this indication.

\section{Materials and Methods}

\subsection{Patients and Treatment}

This retrospective study included all patients with newly diagnosed AML according to 2016 WHO classification [12], excluding acute promyelocytic leukemia, between January 1, 2000 and December 31, 2017. All patients were registered in the Toulouse-Bordeaux DATAML registry. Patients were included in the current study if they were $\geq 18$ years of age, treated by first-line intensive chemotherapy [13], with an FLT3-ITD or FLT3-TKD mutation and an ITD/wild-type (wt) ratio greater than 0.03. Written 
informed consent was obtained in accordance with the Declaration of Helsinki, allowing for the collection of clinical data in the anonymized database. Cytogenetic risk classification was defined according to the Medical Research Council classification [14]. Main salvage regimens used in both centers were based on single-agent cytarabine (high-dose cytarabine, $3 \mathrm{~g} / \mathrm{m}^{2} / 12 \mathrm{~h}$, d1-4; intermediate dose-cytarabine, $1 \mathrm{~g}-1.5 \mathrm{~g} / \mathrm{m}^{2} / 12 \mathrm{~h}, \mathrm{~d} 1-4$ or $\left.1 \mathrm{~g} / \mathrm{m}^{2} / \mathrm{d}, \mathrm{d} 1-5\right)$, combination of an anthracycline plus cytarabine (daunorubicin $60 \mathrm{mg} / \mathrm{m}^{2} / \mathrm{d}, \mathrm{d} 1-3$ or idarubicin $12 \mathrm{mg} / \mathrm{m}^{2} / \mathrm{d}, \mathrm{d} 1-3$ or amsacrine $200 \mathrm{mg} / \mathrm{m}^{2} / \mathrm{d}$, $\mathrm{d} 1-3+$ cytarabine $1.5-3 \mathrm{~g} / \mathrm{m}^{2} / 12 \mathrm{~h}, \mathrm{~d} 1-4$ ), or the FLAG-IDA regimen (fludarabine $30 \mathrm{mg} / \mathrm{m}^{2} / \mathrm{d}, \mathrm{d} 1-5+$ cytarabine $2 \mathrm{~g} / \mathrm{m}^{2} / \mathrm{d}, \mathrm{d} 1-5+$ idarubicin $\left.10 \mathrm{mg} / \mathrm{m}^{2} / \mathrm{d}, \mathrm{d} 1-3+\mathrm{G}-\mathrm{CSF} 5 \mu \mathrm{g} / \mathrm{kg} / \mathrm{d}, \mathrm{d} 1-5\right)$. The choice between these different options was made on a case-by-case basis depending on the patient's performance status, previous treatment history and time to relapse. Gemtuzumab ozogamycin was occasionally used. Sorafenib was also used off-label after preliminary results showing efficacy in FLT3-ITD R/R AML patients [15]. Bone marrow assessment was performed for patients treated by intensive chemotherapy after peripheral recovery or in case of delayed recovery, between days 35 and 45 . Response to treatment, relapse-free survival (RFS), event-free survival (EFS), cumulative incidence of relapse (CIR) and overall survival (OS) were defined according to the European LeukemiaNet 2017 criteria [5]. For R/R AML, OS and EFS were measured from the date of relapse or the date of failure; RFS and CIR were measured from complete remission obtained after salvage therapy. Primary refractory AML was defined as a failure to achieve complete remission (CR) or CR with incomplete hematologic recovery (CRi) after one or two courses of induction chemotherapy; relapse was defined as bone marrow blasts $\geq 5 \%$, reappearance of blasts in the blood or development of extramedullary disease [5].

\subsection{Statistical Analysis}

We described the patients' characteristics at diagnosis and at relapse using numbers and frequencies for qualitative data and using the median, interquartile range (IQR) and range (minimum-maximum) for quantitative data. For survival analyses of RFS, EFS and OS, Kaplan-Meier survival curves were drawn and described using median, IQR and survival at 1, 3 and 5 years. For relapse, cumulative incidence functions were drawn (since nonrelapse mortality was treated as a competing event) and described using CIR at 1, 3 and 5 years. Hazard ratios (HR) and $95 \%$ confidence intervals $(95 \% \mathrm{CI}$ ) were assessed using a standard Cox model for RFS, EFS and OS and using a proportional subdistribution hazard model (an extension of the Cox model) for competing risks for CIR [16]. For the rate of CR or CRi, odds ratios (OR) and $95 \%$ confidence intervals $(95 \% \mathrm{CI})$ were assessed using a standard logistic regression model. Multivariate analyses, for newly diagnosed FLT3-ITD and FLT3-TKD AML patients initially included all potential risk factors (age, performance status (ECOG), AML status (de novo or secondary AML), gender, white blood cells, cytogenetics risk, NPM1 co-mutation status and allogeneic hematopoietic stem cell transplantation (HSCT; only for EFS, RFS, CIR and OS)) associated with endpoints with a $p$-value less than 0.20 in univariate analyses. A stepwise regression was then used to assess variables that were significantly and independently associated with the endpoints ( $p$-value $<0.05)$. The proportional hazard assumption was tested for each covariate of the Cox model using log-log plot method curves and was always supported. When the linear hypothesis was not supported, continuous potential confounding factors were transformed into ordered data. Interactions between variables that were significantly and independently associated with endpoints were tested in final models. None were significant. Allogeneic HSCT was evaluated as a time-dependent covariate. All reported $p$-values were two-sided, and the significance threshold was $<0.05$. Statistical analyses were performed using STATA version 14.2 (STATA Corp., College Station, TX, USA).

\section{Results}

\subsection{Study Population}

Out of 3290 newly diagnosed AML patients included in the DATAML registry between 2000 and 2017, 1453 did not have a recorded status for FLT3 mutation and 364 were not selected to receive 
intensive chemotherapy as a first-line treatment. A total of 347 patients with FLT3-ITD $(n=317)$ or FLT3-TKD $(n=39)$ mutated AML fulfilled the inclusion criteria (Figure S1). Their characteristics are presented in Table 1. One hundred fifty-three patients $(44.1 \%)$ were 60 years of age or older. There were 306 (88.4\%) de novo AML. Extramedullary involvement and leukostasis were observed in 132 (42.7\%) and $53(15.5 \%)$ patients, respectively. The median white blood cell count (WBC) was $52.6 \times 10^{9} / \mathrm{L}(\mathrm{IQR}$ : 20.6-117.8). In FLT3-ITD-mutated patients with documented allelic ratio, 67 out of 141 (47.5\%) had an ITD/wt ratio greater than 0.5 . The vast majority of patients $(N=318,91.6 \%)$ had an intermediate cytogenetic risk (normal karyotype: $N=255 / 311(82 \%)$ ). Two hundred fourteen patients out of 326 $(65.6 \%)$ had an NPM1 co-mutation, and 52 out of 143 patients had a DNMT3A co-mutation (36.4\%).

Table 1. Baseline characteristics of the 347 newly diagnosed FLT3-mutated AML patients treated with intensive chemotherapy.

\begin{tabular}{|c|c|}
\hline Patients' Characteristics & $N=347$ \\
\hline \multicolumn{2}{|l|}{ Age (years) } \\
\hline Median (IQR) & $57.3(47.8-67.6)$ \\
\hline Range & $18.6-81.4$ \\
\hline \multicolumn{2}{|l|}{ Gender: $n(\%)$} \\
\hline Female & $176(50.7)$ \\
\hline Male & $171(49.3)$ \\
\hline \multicolumn{2}{|c|}{ ECOG performance status: $n(\%)$} \\
\hline $0-1$ & $226(73.9)$ \\
\hline$\geq 2$ & $80(26.1)$ \\
\hline \multicolumn{2}{|l|}{ WBC $(\times 109 / \mathrm{L})$} \\
\hline Median (IQR) & $52.6(20.6-117.8)$ \\
\hline Range & $0.4-433.0$ \\
\hline \multicolumn{2}{|l|}{ Tumor burden: $n(\%)$} \\
\hline \multicolumn{2}{|l|}{ Extramedullary involvement } \\
\hline Yes & $137(42.7)$ \\
\hline No & $184(57.3)$ \\
\hline \multicolumn{2}{|l|}{ Leukostasis } \\
\hline Yes & $55(15.5)$ \\
\hline No & $289(84.5)$ \\
\hline \multicolumn{2}{|l|}{$\mathrm{LDH}$} \\
\hline$>$ normal & $311(93.4)$ \\
\hline normal & $22(6.6)$ \\
\hline \multicolumn{2}{|l|}{ Biochemistry: median (IQR) } \\
\hline Creatinine $(\mu \mathrm{mol} / \mathrm{L})$ & $80.0(64.0-101.0)$ \\
\hline Albumin $(\mathrm{g} / \mathrm{L})$ & $36.0(32.0-39.5)$ \\
\hline Fibrinogen (g/L) & $4.0(2.8-5.3)$ \\
\hline \multicolumn{2}{|l|}{ AML status: $n(\%)$} \\
\hline De novo & $306(88.4)$ \\
\hline Secondary AML & $40(11.6)$ \\
\hline \multicolumn{2}{|l|}{ Cytogenetic risk: $n(\%)$} \\
\hline Favorable & $13(3.7)$ \\
\hline Intermediate & 318 (91.6) \\
\hline Normal & $255 / 311(82.0)$ \\
\hline Intermediate-abnormal & $56 / 311(18.0)$ \\
\hline Adverse & $16(4.6)$ \\
\hline
\end{tabular}


Table 1. Cont.

\begin{tabular}{|c|c|}
\hline Patients' Characteristics & $N=347$ \\
\hline \multicolumn{2}{|l|}{ ELN 2010 classification: $n(\%)$} \\
\hline Favorable & $27(8.2)$ \\
\hline Intermediate-1 & $232(70.1)$ \\
\hline Intermediate-2 & $56(16.9)$ \\
\hline Adverse & $16(4.8)$ \\
\hline \multicolumn{2}{|l|}{ FLT3 mutation: $n(\%)$} \\
\hline ITD & $317 / 342(92.7)$ \\
\hline TKD & $39 / 141(27.7)$ \\
\hline \multicolumn{2}{|l|}{ FLT3 ratio ITD/wt: $n(\%)$} \\
\hline $0.03-0.25$ & $34(24.1)$ \\
\hline $0.26-0.50$ & $40(28.4)$ \\
\hline $0.51-0.78$ & $43(30.5)$ \\
\hline$>0.78$ & $24(17.0)$ \\
\hline \multicolumn{2}{|l|}{ NPM1: $n(\%)$} \\
\hline Mutation & $214(65.6)$ \\
\hline No mutation & $112(34.4)$ \\
\hline \multicolumn{2}{|l|}{ IDH1/2 mutations: $n(\%)$} \\
\hline IDH1R132 & $13(7.6)$ \\
\hline IDH2R140 & $9(5.3)$ \\
\hline IDH2R172 & $0(0.0)$ \\
\hline No mutation & $148(87.1)$ \\
\hline \multicolumn{2}{|l|}{ Induction chemotherapy } \\
\hline Daunorubicin-cytarabine & $127(36.6)$ \\
\hline Idarubicin-cytarabine & $101(29.1)$ \\
\hline Idarubicin-cytarabine-lomustine & $103(29.7)$ \\
\hline $\begin{array}{c}\text { Daunorubicin-cytarabine-gemtuzumab } \\
\text { ozogamicin }\end{array}$ & $8(2.3)$ \\
\hline Other & $8(2.3)$ \\
\hline $\begin{array}{l}\text { Allogeneic stem cell transplantation in first } \\
\text { CR: } n(\%)\end{array}$ & $100 / 271(36.9)$ \\
\hline
\end{tabular}

AML: acute myeloid leukemia; CR: complete remission; ELN: European LeukemiaNet; IQR: interquartile range; ITD: internal tandem duplication; LDH: lactate dehydrogenase; TKD: tyrosine kinase domain; WBC: white blood cells count; wt: wild-type.

\subsection{First-Line Treatment and Outcome}

Treatment regimens for induction chemotherapy based on anthracyclines and cytarabine are presented in Table 1. One hundred fifty-one patients $(43.9 \%)$ received hydroxycarbamide as cytoreduction before intensive chemotherapy. Eighty-nine patients $(25.7 \%)$ were admitted to the intensive care unit either during induction therapy or in the first 3 months following the first induction course. Twenty-two patients $(6.3 \%)$ received an FLT3 inhibitor associated with the first induction course: four patients $(1.2 \%)$ received quizartinib or placebo in the QUANTUM-FIRST clinical trial (NCT02668653), and 18 patients (5.2\%) received ponatinib in the PONATINIB-AML clinical trial (NCT02428543). These patients were excluded from the efficacy and survival analyses. Among the 325 patients who received induction chemotherapy without an FLT3 inhibitor, 247 (76.0\%) and 271 $(83.4 \%)$ achieved CR/CRi after one or two courses, respectively, whereas 26 patients $(8.0 \%)$ failed to achieve a response. Early death rate by day 30 was $8.6 \%(n=28)$. Allogeneic stem cell transplantation was performed in first CR in 100 patients (36.9\%).

After a median follow-up of 69.9 months (IQR: 42.1-116.1), 149 out of 271 (55.0\%) patients in CR/CRi relapsed. The CIR was 39.0\% (95\%CI: 34.0-45.0), 52.0\% (95\%CI: 46.0-58.0) and 57.0\% (95\%CI: 50.0-63.0) at 1, 3 and 5 years, respectively. The median RFS, EFS and OS were 13.6 (IQR: 5.7-154.0), 11.3 (IQR: 5.1-85.8) and 17.5 (IQR: 8.2-115.2) months, respectively (Figure 1, Table 2). Multivariate analyses 
showed that age $\geq 60$ years (adjusted hazard ratio (aHR) 1.70 (95\%CI: 1.29-2.24), $p<0.001$ ), female gender (aHR 0.72 (95\%CI: 0.54-0.94), $p=0.017$ ), performance status $\geq 2$ (aHR 1.86 (95\%CI: 1.36-2.55), $p<0.001$ ) and favorable cytogenetics (aHR 0.16 (95\%CI: 0.04-0.65), $p=0.011$ ) were significantly and independently associated with OS (Table S1). Multivariate analyses for factors associated with CR/CRi, RFS, CIR and EFS are presented in the Supplementary Data (Tables S2-S5).

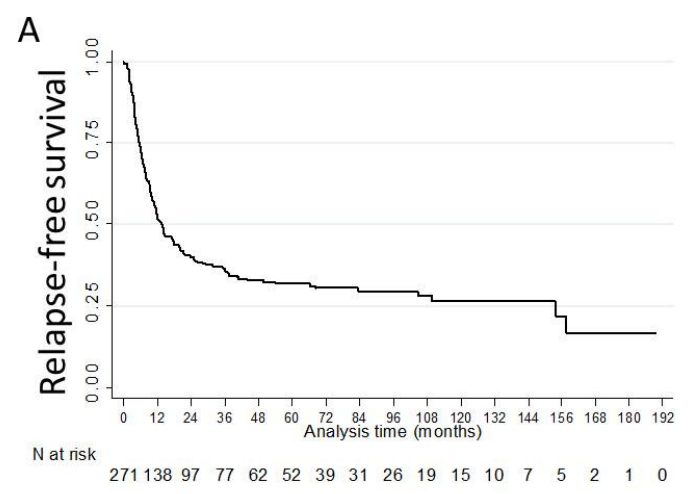

B

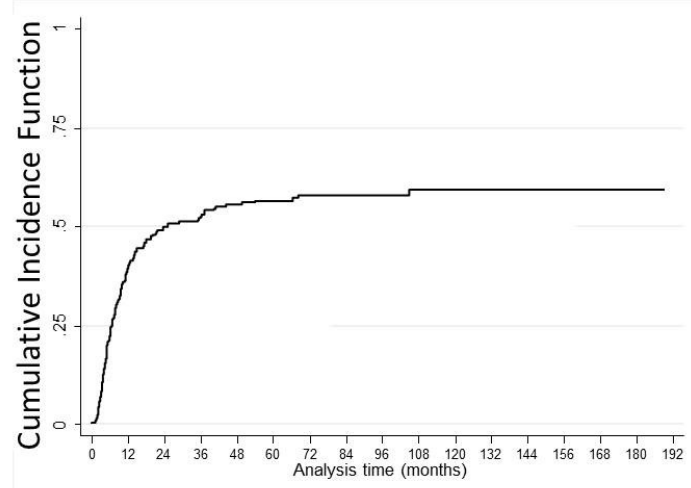

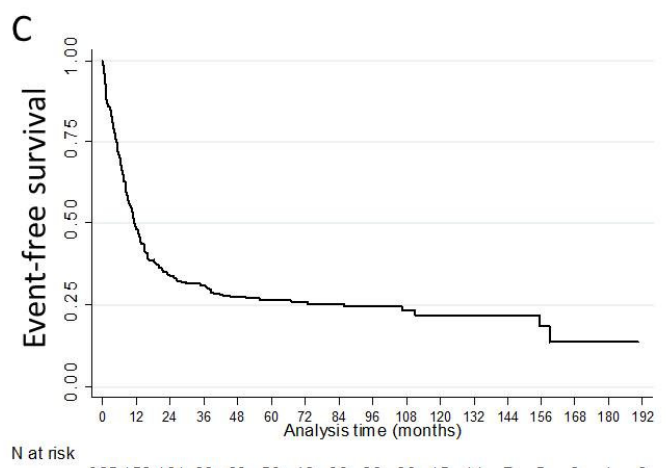

D

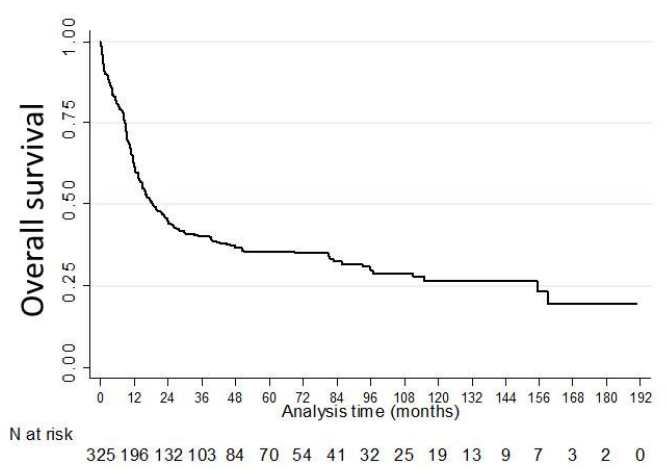

Figure 1. Outcome of patients with newly diagnosed FLT3-mutated acute myeloid leukemia: (A) relapse-free survival; (B) cumulative incidence of relapse; (C) event-free survival; (D) overall survival.

Table 2. Survival of FLT3-mutated acute myeloid leukemia after first-line FLT3 inhibitor-free intensive chemotherapy.

\begin{tabular}{cccccc}
\hline Endpoint & $\mathbf{N}$ & $\begin{array}{c}\text { Median (Months, } \\
\text { (IQR)) }\end{array}$ & $\begin{array}{c}\mathbf{1 - Y e a r} \mathbf{\%} \\
\mathbf{( 9 5 \% C I )}\end{array}$ & $\begin{array}{c}\mathbf{3 - Y e a r} \% \\
\mathbf{( 9 5 \% C I )}\end{array}$ & $\begin{array}{c}\mathbf{5 - Y e a r} \mathbf{\%} \\
\mathbf{( 9 5 \% C I )}\end{array}$ \\
\hline RFS & 271 & $13.6(5.7-154.0)$ & $52.7(46.5-58.5)$ & $36.3(30.4-42.1)$ & $31.8(26.1-37.7)$ \\
CIR & 271 & & $39.0(34.0-45.0)$ & $52.0(46.0-58.0)$ & $57.0(50.0-63.0)$ \\
EFS & 325 & $11.3(5.1-85.8)$ & $48.0(42.4-53.3)$ & $31.0(25.9-36.2)$ & $26.5(21.6-31.6)$ \\
OS & 325 & $17.5(8.2-115.2)$ & $62.0(56.4-67.0)$ & $40.1(34.6-45.5)$ & $35.5(30.0-41.0)$ \\
\hline
\end{tabular}

CI: confidence interval; CIR: cumulative incidence of relapse; EFS: event-free survival; IQR: interquartile range; RFS: relapse-free survival; OS: overall survival.

\subsection{Characteristics of Relapsed or Refractory FLT3-Mutated AML}

In this series, there were 186 relapses, of which 12 (6.5\%) were FLT3-wt at relapse and were therefore excluded from analyses. Thus, the total number of R/R patients was 174 (48 primary induction failure $(27.6 \%)$ and 126 relapses $(72.4 \%))$. Among these 126 relapsed patients, 48 patients $(27.6 \%)$ relapsed within the first 6 months after CR/CRi and 78 (44.8\%) relapsed after 6 months. Among these 126 patients, $29(23.0 \%)$ relapsed after allogeneic HSCT. The median time to relapse was 7.7 months (IQR: 4.7-12.6). Among the 48 refractory patients, 12 (25.0\%) and 36 (75.0\%) received one or two 
induction courses, respectively. The characteristics of these 174 R/R FLT3-mutated AML patients are shown in Table 3.

Table 3. Characteristics of patients with relapsed/refractory FLT3-mutated acute myeloid leukemia $(n=174)$.

\begin{tabular}{|c|c|}
\hline Patients' Characteristics & $\begin{array}{c}\text { R/R FLT3-Mutated AML } \\
\qquad N=174\end{array}$ \\
\hline Age (years): & \\
\hline Refractory: Median (IQR) & $57.8(43.1-67.3)$ \\
\hline Relapse: Median (IQR) & $59.9(47.2-70.7)$ \\
\hline \multicolumn{2}{|l|}{ Gender: $n(\%)$} \\
\hline Female & $88(50.6)$ \\
\hline Male & $86(49.4)$ \\
\hline \multicolumn{2}{|l|}{ ECOG performance status: $n(\%)$} \\
\hline $0-1$ & $106(79.7)$ \\
\hline$\geq 2$ & $27(20.3)$ \\
\hline \multicolumn{2}{|l|}{ Status: $n(\%)$} \\
\hline Refractory & $48(27.6)$ \\
\hline One induction course & $12(6.9)$ \\
\hline Two induction courses & $36(20.7)$ \\
\hline Relapse & $126(72.4)$ \\
\hline$<6$ months & $48(27.6)$ \\
\hline$\geq 6$ months & $78(44.8)$ \\
\hline \multicolumn{2}{|l|}{ Duration of CR/CRi before relapse (months): } \\
\hline Median (IQR) & $7.7(4.7-12.6)$ \\
\hline Previous allogeneic HSCT in first CR: $n(\%)$ & $29(23.0)$ \\
\hline \multicolumn{2}{|l|}{ FLT3 ITD/wt ratio $(N=65)(\%)$ : } \\
\hline Median (IQR) & $50.0(28.0-68.0)$ \\
\hline \multicolumn{2}{|l|}{ Co-mutations: $n(\%)$} \\
\hline \multicolumn{2}{|l|}{ NPM1 mutations } \\
\hline Yes & $94(56.6)$ \\
\hline No & $72(43.4)$ \\
\hline \multicolumn{2}{|l|}{ DNMT3A mutations } \\
\hline Yes & $20(29.9)$ \\
\hline No & $47(70.1)$ \\
\hline \multicolumn{2}{|l|}{ CEBPA mutations } \\
\hline Yes & $6(7.4)$ \\
\hline No & $75(92.6)$ \\
\hline \multicolumn{2}{|l|}{ IDH1/2 mutations } \\
\hline Yes & $8(9.4)$ \\
\hline No & $77(90.6)$ \\
\hline \multicolumn{2}{|l|}{ N/K RAS mutations } \\
\hline Yes & $3(8.6)$ \\
\hline No & $32(91.4)$ \\
\hline \multirow{2}{*}{\multicolumn{2}{|c|}{$\begin{array}{c}\text { WBC }(\times 109 / \mathrm{L}): \\
\text { At diagnosis (refractory) }\end{array}$}} \\
\hline & \\
\hline Median (IQR) & $72.8(18.3-149.8)$ \\
\hline Range & $0.6-317.0$ \\
\hline \multicolumn{2}{|l|}{ At relapse } \\
\hline Median (IQR) & $7.4(3.4-26.9)$ \\
\hline Range & $0.1-436.0$ \\
\hline
\end{tabular}

CR: complete remission; CRi: CR with incomplete hematological recovery; HSCT: hematologic stem cell transplantation; IQR: interquartile range; WBC: white blood cells count; wt: wild-type. 


\subsection{Treatment of Relapsed or Refractory FLT3-Mutated AML}

One hundred twenty-nine patients $(74.1 \%)$ received a salvage therapy: $41(31.8 \%)$ received I/HDAC-based salvage chemotherapy; 48 (37.2\%) received an anthracycline-based chemotherapy (either " $7+3$ "-like or combined to I/HDAC); 10 (7.8\%) received a GO-based salvage chemotherapy, $6(4.7 \%)$ received azacitidine and $3(2.3 \%)$ received LDAC. Five patients $(3.9 \%)$ received another type of treatment (radiotherapy for extramedullary localization, dactinomycin or all-trans retinoic acid). Twenty-five patients (19.4\%) received a FLT3 inhibitor-containing regimen; 11 patients received gilteritinib or quizartinib as a single agent, one patient received midostaurin with dactinomycin, and 13 patients received sorafenib alone or associated to dactinomycin, LDAC or all-trans retinoic acid. In total, 99 patients $(76.7 \%)$ received high-intensity chemotherapy whereas nine patients $(7.0 \%)$ received azacitidine or LDAC and 17 patients (13.2\%) received low-intensity chemotherapy. Finally, four patients (3.1\%) received allogeneic hematopoietic stem cell transplantation (HSCT) after sequential conditioning without previous salvage chemotherapy. Forty-five patients (25.9\%) received best supportive care only (Figure S1).

The 11 patients who received gilteritinib or quizartinib as a single agent or midostaurin with dactinomycin were excluded from efficacy analyses, whereas those receiving sorafenib were included among the low-intensity regimens because this treatment was considered "as a compassionate real-world approach" in this setting. Three more patients were also excluded because of loss of follow-up. In total, 114 patients (32 refractory $(28.1 \%)$ and 82 relapses $(71.9 \%)$ ) received salvage treatment other than quizartinib, gilteritinib and midostaurin. CR/CRi was achieved in 57 patients (50.0\%). Fifty-two patients (45.6\%) failed to achieve CR/CRi and the five remaining patients $(4.4 \%)$ died before evaluation. Thirty-nine patients (34.2\%) could proceed to allogeneic HSCT.

\subsection{Outcome of Relapsed or Refractory FLT3-Mutated AML}

The 30-day and 60-day death rates were 6.1\% $(n=7)$ and $14.9 \%(n=17)$. Median duration of response in primary refractory and relapsed patients were 4.2 (IQR: 3.3-12.0) and 5.7 (IQR: 2.4-9.7) months, respectively. With a median follow-up of 63.9 months (IQR: 34.6-130.0), the median RFS, EFS and OS were 6.8 (IQR: 3.6-85.6), 3.4 (IQR: 1.3-10.6) and 8.2 (IQR: 3.0-32.0) months, respectively (Figure 2, Table 4). Multivariate analyses for factors significantly and independently associated with the various endpoints were not sufficiently powered to draw any conclusion [17]. In univariate analyses, FLT3 ratio ITD/wt $(\leq 0.78$ versus $>0.78)$ was not significantly associated with RFS $(p=0.5893)$, EFS $(p=0.6131)$, OS $(p=0.6758)$ or second response $(p=0.4160)$.

Table 4. Survival of relapsed/refractory FLT3-mutated AML after salvage treatment.

\begin{tabular}{|c|c|c|c|c|c|}
\hline & $\mathbf{N}$ & $\begin{array}{c}\text { Median } \\
\text { (Months, (IQR)) }\end{array}$ & $\begin{array}{c}\text { 1-Year } \\
\%(95 \% \mathrm{CI})\end{array}$ & $\begin{array}{c}\text { 3-Year } \\
\%(95 \% \mathrm{CI})\end{array}$ & $\begin{array}{c}5-\text {-Year } \\
\%(95 \% \mathrm{CI})\end{array}$ \\
\hline RFS & 57 & $6.8(3.6-85.6)$ & $42.9(29.8-55.3)$ & $31.2(19.4-43.7)$ & $31.2(19.4-43.7)$ \\
\hline EFS & 114 & $3.4(1.3-10.6)$ & $22.4(15.2-30.4)$ & $15.4(9.3-22.9)$ & $15.4(9.3-22.9)$ \\
\hline OS & 114 & $8.2(3.0-32.0)$ & $36.0(27.2-44.8)$ & $24.7(17.0-33.3)$ & $19.7(12.5-28.2)$ \\
\hline
\end{tabular}

CI: confidence interval; CIR: cumulative incidence of relapse; EFS: event-free survival; IQR: interquartile range; RFS: relapse-free survival; OS: overall survival. 
A
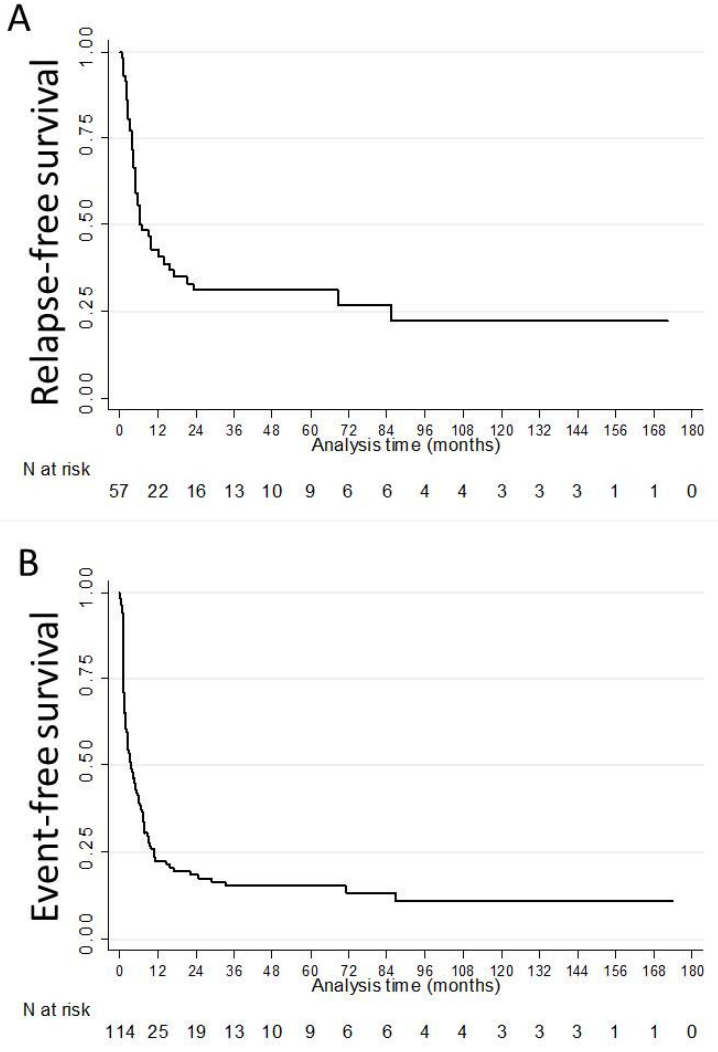

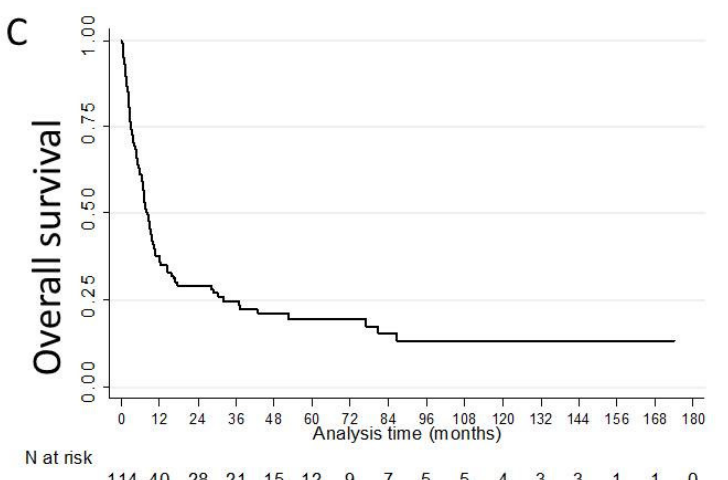

$\begin{array}{llllllllllllllll}114 & 40 & 28 & 21 & 15 & 12 & 9 & 7 & 5 & 5 & 4 & 3 & 3 & 1 & 1 & 0\end{array}$

Figure 2. Outcome of patients with refractory or relapsed FLT3-mutated acute myeloid leukemia:

(A) relapse-free survival; (B) event-free survival; (C) overall survival.

\section{Discussion}

Most AML patients refractory to standard induction chemotherapy or relapsing after complete response die from disease progression. A longer relapse-free interval after first $C R$, presence of a CBF-AML at diagnosis, lower age at relapse and no previous stem-cell transplantation during first-line therapy are factors associated with more favorable prognosis in this setting but concern a minority of patients [18]. Moreover, FLT3-ITD is an independent poor prognostic factor in R/R AML patients when treated by intensive chemotherapy at time of relapse or refractory disease [19]. This has been confirmed in two randomized phase 3 trials for FLT3-mutated AML assessing FLT3 inhibitors versus standard of care, in which results of control arms were particularly poor $[8,10]$.

In the ADMIRAL trial for patients with R/R FLT3-mutated AML, single-agent gilteritinib was found to significantly improve the median OS from 5.6 to 9.3 months compared with the conventional care regimen. Here, we examined the characteristics and outcome of R/R FLT3-mutated AML patients included in the Toulouse-Bordeaux DATAML registry, in order to evaluate the efficacy of standard treatments that appeared to have limited efficacy in the ADMIRAL study. Our study reflects routine practice in a registry that covers a region of 6 million inhabitants (approximately $10 \%$ of the French population). One potential source of bias in the current study is FLT3 status, which was known in 56\% of all patients added to the DATAML registry between 2000 and 2017, especially in the early 2000s. However, the FLT3 status is known in $66 \%$ of patients selected for intensive chemotherapy and, among them, for $81 \%$ of patients with intermediate cytogenetic risk. Moreover, because FLT3-ITD but not FLT3-TKD had a long-lasting prognostic value, FLT3-TKD has not been systematically monitored until recently with the advent of FLT3 inhibitors such as midostaurin, approved for first-line therapy in 2017. Therefore, FLT3-TKD patients are likely underrepresented in our study. Also, there may be measurement bias in the comparison of the remission rates based on real-world data versus a prospective trial, but this measurement bias obviously does not apply to OS or to the allogeneic HSCT 
rate, which are objective endpoints. The main patient characteristics were roughly similar between the salvage chemotherapy group of ADMIRAL and our cohort, apart from a slightly younger median age. This could partly explain the differences in treatment allocation, as $20.2 \%$ of patients received a low-intensity regimen as salvage treatment in our study (among them, one third received LDAC or azacitidine), whereas $39.5 \%$ of patients were allocated to LDAC or azacitidine in the ADMIRAL control arm. The rate of CR or CRi in our study was $50.0 \%$ compared to $15.3 \%$ in the standard arm of the ADMIRAL study (16\% CR patients treated with high intensity chemotherapy). Moreover, the transplantation rate in our study $(34.2 \%)$ was higher than in the standard arm of the ADMIRAL trial $(15 \%)$. Thus, the higher response and transplantation rates may explain the better outcome observed in our cohort compared to the control arm of the ADMIRAL trial.

Obviously, our results do not mean that real-world treatments are equivalent to those of gilteritinib. For example, ADMIRAL patients preselected for high-intensity chemotherapy and randomized to receive gilteritinib had a median OS of 10.5 months, indicating that the way patients are selected for a given treatment influences outcome.

The adverse effects of salvage treatments were not specifically addressed in this study because it is well established that high-intensity regimens, including high-dose cytarabine, FLAG-IDA regimen or equivalents, are very toxic in terms of use of healthcare resources, length of hospital stay, transfusion support, infections and quality of life. In the ADMIRAL study, 30-day and 60-day mortality were $2.0 \%$ and $7.7 \%$ in the gilteritinib arm compared to $10.2 \%$ and $19.0 \%$ in the control arm or $6.1 \%$ and $14.9 \%$ in our study. Quality of life could also be improved with this oral targeted agent compared to high-intensity chemotherapy; this makes sense but is not clearly demonstrated to date.

\section{Conclusions}

Given the underperforming results of second-line therapeutic strategies, the treatment of R/R FLT3-mutated AML patients remains a major challenge. Our study shows that some patients could still benefit from intensive chemotherapy (i.e., achieving a CR while maintaining a suitable performance status to allow transplantation), although we failed (because of too small sample size) to identify factors that could help identify patients who will benefit. This suggests that using combinations or sequential treatments based on gilteritinib and chemotherapy could improve results. Obviously, combinations of targeted therapies such as FLT3 inhibitors and BCL2 inhibitors, for example, are also promising options for R/R FLT3-mutated AML patients [20].

Supplementary Materials: The following are available online at http://www.mdpi.com/2072-6694/12/4/773/s1, Figure S1: Flowchart, Table S1: Cox model for factors independently associated with overall survival among FLT3-mutated AML patients, Table S2: Logistic regression model for factors independently associated with CR/CRi among FLT3-mutated AML patients, Table S3: Cox model for factors independently associated with relapse-free survival among FLT3-mutated AML patients, Table S4: Cox model for factors independently associated with cumulative incidence of relapse among FLT3-mutated AML patients, Table S5: Cox model for factors independently associated with event-free survival among FLT3-mutated AML patients.

Author Contributions: Conceptualization, S.B., P.-Y.D., C.R. and A.P.; Data curation, S.B., P.-Y.D., N.G., H.L., A.S. and A.-C.D.G.; Formal analysis, L.L., A.B., E.D., J.-B.R., J.-P.V., F.V., N.L., I.L. and E.K.; Investigation, S.B.; Methodology, E.B.; Supervision, S.B., P.-Y.D. and C.R.; Writing-original draft, S.B., P.-Y.D., S.T. and C.R.; Writing-review \& editing, S.B., P.-Y.D., E.B., L.L., A.B., E.D., S.T., N.G., T.L., H.L., J.-B.R., J.-P.V., F.V., J.-P.V., I.L., E.K., A.S., A.-C.D.G., C.R. and A.P; Final approval: all authors. All authors have read and agreed to the published version of the manuscript.

Funding: This research received no external funding.

Acknowledgments: We would like to thank the data management unit of Toulouse University for its support enabling e-CRF. We thank all the members of the G.A.E.L (Gaël Adolescent Espoir Leucémie) association and the FONROGA foundation.

Conflicts of Interest: CR has received research grants from Amgen, Novartis, Celgene, Jazz Pharmaceuticals, Agios, Chugai, Sunesis, Astellas, and MaatPharma and is an advisor for AbbVie, Sunesis, Janssen, Jazz, Novartis, Celgene, Astellas, Daiichi-Sankyo, Macrogenics, and Pfizer. ED is advisor for Novartis. SB is an advisor for Jazz, Daiichi-Sankyo, Sanofi, and Astellas. PYD is an advisor for Daiichi-Sankyo, Astellas. AP has received grants from 
Roche, Celgene, Sunesis, Novartis, Jazz, AbbVie, Astellas, Daiichi-Sankyo and is an advisor for Roche, Celgene, Sunesis, Amgen, Novartis, Jazz, AbbVie, Astellas, Daiichi-Sankyo, Sanofi, Pfizer.

\section{References}

1. Small, D.; Levenstein, M.; Kim, E.; Carow, C.; Amin, S.; Rockwell, P.; Witte, L.; Burrow, C.; Ratajczak, M.Z.; Gewirtz, A.M.; et al. STK-1, the human homolog of Flk-2/Flt-3, is selectively expressed in CD34+ human bone marrow cells and is involved in the proliferation of early progenitor/stem cells. Proc. Natl. Acad. Sci. USA 1994, 91, 459-463. [CrossRef] [PubMed]

2. Papaemmanuil, E.; Gerstung, M.; Bullinger, L.; Gaidzik, V.I.; Paschka, P.; Roberts, N.D.; Potter, N.E.; Heuser, M.; Thol, F.; Bolli, N.; et al. Genomic Classification and Prognosis in Acute Myeloid Leukemia. N. Engl. J. Med. 2016, 374, 2209-2221. [CrossRef] [PubMed]

3. Nakao, M.; Yokota, S.; Iwai, T.; Kaneko, H.; Horiike, S.; Kashima, K.; Sonoda, Y.; Fujimoto, T.; Misawa, S. Internal tandem duplication of the flt3 gene found in acute myeloid leukemia. Leukemia 1996, 10, 1911-1918. [PubMed]

4. Kiyoi, H.; Naoe, T.; Yokota, S.; Nakao, M.; Minami, S.; Kuriyama, K.; Takeshita, A.; Saito, K.; Hasegawa, S.; Shimodaira, S.; et al. Internal tandem duplication of FLT3 associated with leukocytosis in acute promyelocytic leukemia. Leukemia Study Group of the Ministry of Health and Welfare (Kohseisho). Leukemia 1997, 11, 1447-1452. [CrossRef] [PubMed]

5. Döhner, H.; Estey, E.; Grimwade, D.; Amadori, S.; Appelbaum, F.R.; Büchner, T.; Dombret, H.; Ebert, B.L.; Fenaux, P.; Larson, R.A.; et al. Diagnosis and management of AML in adults: 2017 ELN recommendations from an international expert panel. Blood 2017, 129, 424-447. [CrossRef] [PubMed]

6. Smith, C.C.; Wang, Q.; Chin, C.S.; Salerno, S.; Damon, L.E.; Levis, M.J.; Perl, A.E.; Travers, K.J.; Wang, S.; Hunt, J.P.; et al. Validation of ITD mutations in FLT3 as a therapeutic target in human acute myeloid leukaemia. Nature 2012, 485, 260-263. [CrossRef]

7. Stone, R.M.; Mandrekar, S.J.; Sanford, B.L.; Laumann, K.; Geyer, S.; Bloomfield, C.D.; Thiede, C.; Prior, T.W.; Döhner, K.; Marcucci, G.; et al. Midostaurin plus Chemotherapy for Acute Myeloid Leukemia with a FLT3 Mutation. N. Engl. J. Med. 2017, 377, 454-464. [CrossRef] [PubMed]

8. Perl, A.E.; Martinelli, G.; Cortes, J.E.; Neubauer, A.; Berman, E.; Paolini, S.; Montesinos, P.; Baer, M.R.; Larson, R.A.; Ustun, C.; et al. Gilteritinib or Chemotherapy for Relapsed or Refractory FLT3-Mutated AML. N. Engl. J. Med. 2019, 381, 1728-1740. [CrossRef] [PubMed]

9. Cortes, J. Crenolanib besylate, a type I pan-FLT3 inhibitor, to demonstrate clinical activity in multiply relapsed FLT3-ITD and D835 AML. In Oral Abstract \#7008; AACR: Chicago, IL, USA, 2016.

10. Cortes, J.E.; Khaled, S.; Martinelli, G.; Perl, A.E.; Ganguly, S.; Russell, N.; Krämer, A.; Dombret, H.; Hogge, D.; Jonas, B.A.; et al. Quizartinib versus salvage chemotherapy in relapsed or refractory FLT3-ITD acute myeloid leukaemia (QuANTUM-R): A multicentre, randomised, controlled, open-label, phase 3 trial. Lancet Oncol. 2019, 20, 984-997. [CrossRef]

11. Megías-Vericat, J.E.; Martínez-Cuadrón, D.; Sanz, M.A.; Montesinos, P. Salvage Regimens Using Conventional Chemotherapy Agents for Relapsed/Refractory Adult AML Patients: A Systematic Literature Review. Ann. Hematol. 2018, 97, 1115-1153. [CrossRef]

12. Arber, D.A.; Orazi, A.; Hasserjian, R.; Thiele, J.; Borowitz, M.J.; Le Beau, M.M.; Bloomfield, C.D.; Cazzola, M.; Vardiman, J.W. The 2016 revision to the World Health Organization classification of myeloid neoplasms and acute leukemia. Blood 2016, 127, 2391-2405. [CrossRef] [PubMed]

13. Bertoli, S.; Tavitian, S.; Huynh, A.; Borel, C.; Guenounou, S.; Luquet, I.; Delabesse, E.; Sarry, A.; Laurent, G.; Attal, M.; et al. Improved outcome for AML patients over the years 2000-2014. Blood Cancer J. 2017, 7, 635. [CrossRef] [PubMed]

14. Grimwade, D.; Walker, H.; Oliver, F.; Wheatley, K.; Harrison, C.; Harrison, G.; Rees, J.; Hann, I.; Stevens, R.; Burnett, A.; et al. The importance of diagnostic cytogenetics on outcome in AML: Analysis of 1612 patients entered into the MRC AML 10 trial. The Medical Research Council Adult and Children's Leukaemia Working Parties. Blood 1998, 92, 2322-2333. [CrossRef] [PubMed]

15. Metzelder, S.K.; Michel, C.; von Bonin, M.; Rehberger, M.; Hessmann, E.; Inselmann, S.; Solovey, M.; Wang, Y.; Sohlbach, K.; Brendel, C.; et al. NFATc1 as a therapeutic target in FLT3-ITD-positive AML. Leukemia 2015, 29, 1470-1477. [CrossRef] [PubMed] 
16. Fine, J.; Gray, R. A Proportional Hazards Model for the Subdistribution of a Competing Risk. J. Am. Stat. Assoc. 1999, 94, 496-509. [CrossRef]

17. Concato, J.; Feinstein, A.R.; Holford, T.R. The risk of determining risk with multivariable models. Ann. Int. Med. 1993, 118, 201-210. [CrossRef]

18. Breems, D.A.; Van Putten, W.L.; Huijgens, P.C.; Ossenkoppele, G.J.; Verhoef, G.E.; Verdonck, L.F.; Vellenga, E.; De Greef, G.E.; Jacky, E.; Van der Lelie, J.; et al. Prognostic index for adult patients with acute myeloid leukemia in first relapse. J. Clin. Oncol. 2005, 23, 1969-1978. [CrossRef]

19. Chevallier, P.; Labopin, M.; Turlure, P.; Prebet, T.; Pigneux, A.; Hunault, M.; Filanovsky, K.; Cornillet-Lefebvre, P.; Luquet, I.; Lode, L.; et al. A new Leukemia Prognostic Scoring System for refractory/relapsed adult acute myelogeneous leukaemia patients: A GOELAMS study. Leukemia 2011, 25, 939-944. [CrossRef] [PubMed]

20. Perl, A.E.; Daver, N.G.; Pratz, K.W.; Maly, J.; Hong, W.-J.; Bahceci, E.; Tong, B.; Tian, T.; Dilley, K. Venetoclax in Combination with Gilteritinib in Patients with Relapsed/Refractory Acute Myeloid Leukemia: A Phase 1b Study. Blood 2019, 134, 3910-3911. [CrossRef]

(C) 2020 by the authors. Licensee MDPI, Basel, Switzerland. This article is an open access article distributed under the terms and conditions of the Creative Commons Attribution (CC BY) license (http://creativecommons.org/licenses/by/4.0/). 